\title{
STRUCTURAL ANALYSIS OF THE ITER VACUUM VESSEL FROM DISRUPTION LOADING WITH HALO ASYMMETRY*
}

\section{$\operatorname{CONF}-9606 / 16--52$}

Bernard W. Riemer

Oak Ridge National Laboratory

P. O. Box 2008, MS-6332

Oak Ridge, TN 37831

(423) $574-6502$

\author{
Royce O. Sayer \\ Oak Ridge National Laboratory \\ P. O. Box 2008, MS-6418 \\ Oak Ridge, TN 37831 \\ (423) 574-4755
}

\section{ABSTRACT}

Static structural analyses of the ITER vacuum vessel were performed with toroidally asymmetric disruption loads. Asymmetric halo current conditions were assumed to modify symmetric disruption loads which resulted in net lateral loading on the vacuum vessel torus. Structural analyses with the asymmetric loading indicated significantly higher vessel stress and blanket support forces than with symmetric disruption loads. A recent change in the vessel support design which provided toroidal constraints at each mid port was found to be effective in reducing torus lateral movement and vessel stress.

\section{INTRODUCTION}

Toroidal asymmetries in halo currents have been observed during plasma disruptions in tokamak experiments, and recently have been a subject of intense study. Such asymmetries induce peak loading in limited toroidal regions of structure near the plasma and result in net lateral loading on the vacuum vessel torus. Full advantage can no longer be taken of the structural symmetry typical in tokamaks when performing an analysis of an asymmetric disruption. Static structural analyses of postulated asymmetric conditions using loads derived from the Tokamak Simulation Code (TSC) ${ }^{1}$ have been performed on the International Thermonuclear Experimental Reactor (ITER) vacuum vessel and blanket / shield assembly by using cyclic symmetry ${ }^{2}$, a means of using the structural symmetry with non-symmetric loads.

TSC simulations include halo current effects and electromagnetic loads. Although they have an axisymmetric basis, TSC loads have been mapped to three dimensional structural models of a segment of the ITER vacuum vessel and used for dynamic and static analyses ${ }^{3,4}$. For this work the segment's structural forces were separated into two groups; those caused by toroidal current and those by poloidal current, the latter being primarily due to halo current. Halo asymmetry conditions were postulated based on experimental observations, and the poloidal current force group was modified accordingly. For example, if halo current varied as $(1-\cos \theta)$ around the torus the same distribution would be applied to the poloidal current force group.

Only one toroidal segment is explicitly defined in the finite element model, but rotated virtual segments are used and loaded separately in the structural analysis. ITER has 20 segments. In each the two load groups are recombined with poloidal current loads scaled by the distribution factor corresponding to the respective segment. The particular TSC simulation used was a slow vertical disruption event (VDE) where halo effects were relatively large.

\section{DISRUPTION LOAD}

The TSC simulation of the slow VDE used ${ }^{5}$ for these analyses had thermal quench occurring at $1.30 \mathrm{~s}$. The forces induced in one $18^{\circ}$ segment, without asymmetry, are summarized in Fig. 1.

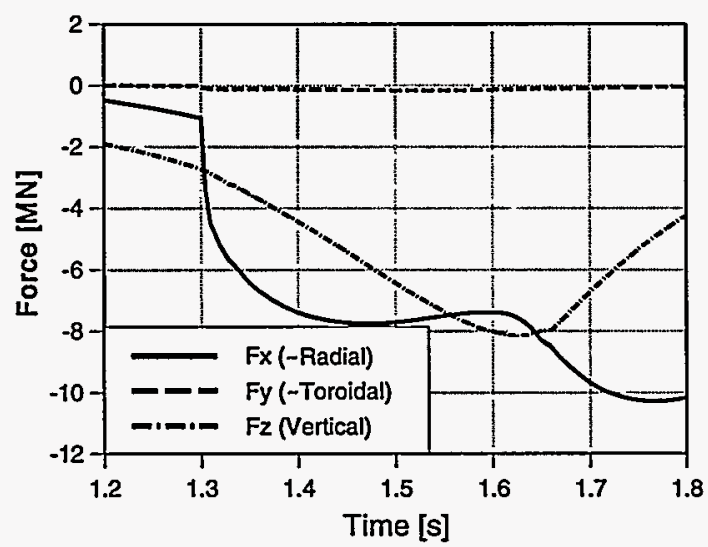

Fig. 1 Slow VDE Total Force for $18^{\circ}$ Vessel Segment

Since the structural analyses were to be done statically, a specific disruption time point had to be chosen. Forces were processed to show induced moments about the machine axis separately considering the inner blanket, outer blanket and vessel. The moments peak at $1.560 \mathrm{~s}$ as seen in Fig. 2 , and from this was inferred a peak halo effect. 


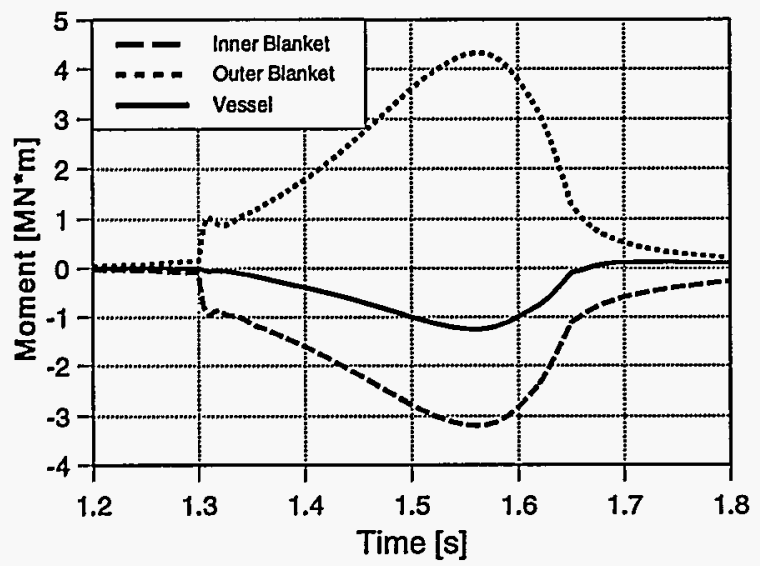

Fig. 2 Moments About Machine Axis for $18^{\circ}$ Vessel Segment

Forces due to poloidal currents are shown on an elevation view of a single shell model in Fig. 3 (with vessel supports removed). The largest forces act on the inboard blanket in the radial direction. In plan view these forces have a small out of plane component. By contrast toroidal current forces act entirely in the $\mathrm{RZ}$ plane. Forces acting on the vessel are small in Fig. 3, with the exception of the lower vessel region. Because a divertor mesh was not included in the model, TSC divertor forces were mapped onto the closest vessel nodes, thus causing the increase in this vessel region.

\section{STRUCTURAL MODELS}

Two finite element models were employed for the structural analyses, both of which had been used for analysis with symmetric loading. The first had been developed for dynamic analyses and used a single shell of elements to model the double wall vacuum vessel with equivalent composite properties. This reduced degrees of freedom for the long running dynamic simulations. The blankets were included with a coarse mesh to give a reasonable representation of their stiffness, inertia and loading, which in turn provided better loading to the vessel at the blanket supports. The second, used for a single case only, was a more detailed model of the vessel which featured both vessel walls and ribs, but did not include blankets. Loads from the blankets were applied to this model at support attachment points which were obtained from the corresponding single shell analysis.

The single shell vessel model had several features which also made it attractive for asymmetric loading. Although the vessel was of primary importance, including the blankets and their supports in the model gives more accurate support loading on the vessel than if blanket forces were applied directly to the vessel at the support attachment points. The asymmetric static analyses benefit from the reduced degrees

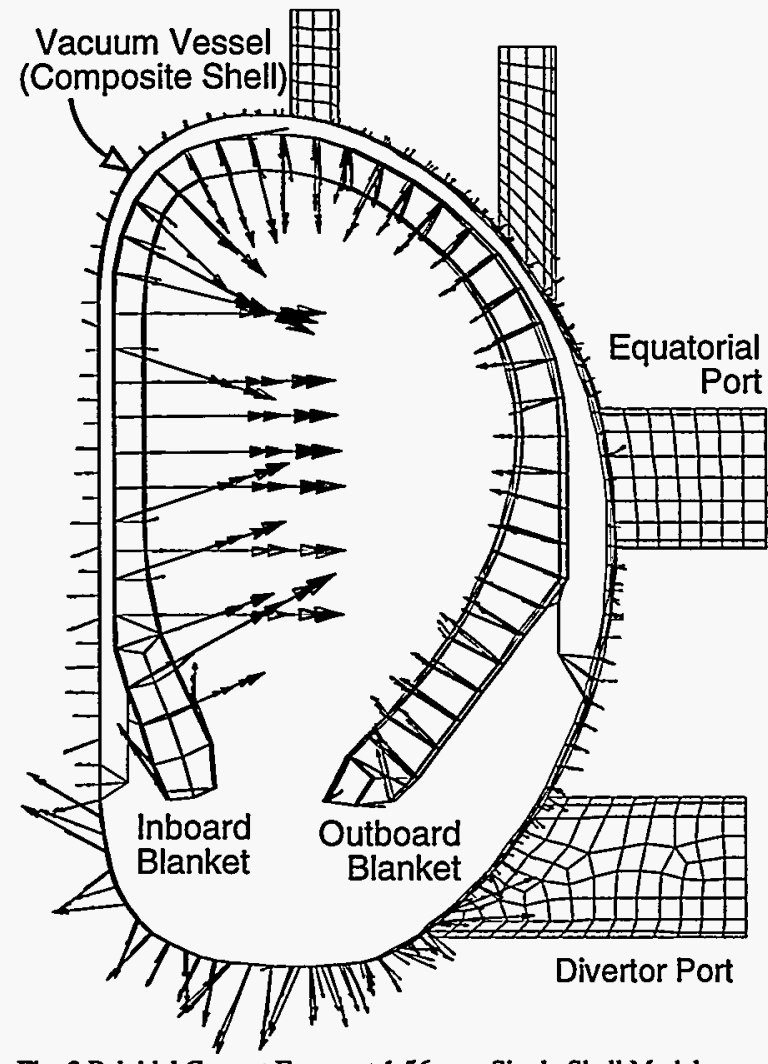

Fig. 3 Poloidal Current Forces at $1.56 \mathrm{~s}$ on Single Shell Model

of freedom since they also use considerable computer resources. Obtaining blanket support forces and moments provides valuable design information and these loads can be used on the double wall vessel model without blankets.

At the time these analyses were begun the baseline vessel support was a truss type structure nearly $6 \mathrm{~m}$ in height which connected thick plate extensions from the base of the vessel to ground. At ground were pin connections which fixed all translations but allowed rotations; at the plate connection only rotation about a toroidal axis was free. Preliminary seismic analysis of this configuration indicated potential difficulties which would also be manifested in asymmetric disruptions because in both cases the torus would have net lateral loading. In symmetric disruptions the lateral forces in each segment cancel out when added over the torus, and no problems particular to this deficiency had been observed.

Later development of an alternative vessel support to address the lack of lateral torus support began. This configuration hung the vessel from the toroidal field (TF) coil structure via assemblies of flexible plates; the assemblies provide little radial or toroidal stiffness. A toroidal constraint is added at each equatorial port via linkage to the TF structure thus providing lateral support to the torus. Incorporating this configuration into the single shell model was straightforward, so a comparison to the truss support could be directly made. 
The double wall model was only analyzed with the truss type support under one asymmetry case. Blanket support forces and moments from the corresponding single shell analysis were used to load this model's blanket attachment points. Disruption loads were not available as separate poloidal current and toroidal current induced groups, so the complete load set was applied equally to all torus segments of the vessel. Only the blanket loads varied segment to segment. This should be adequate since essentially all the halo current loads occur in the blankets and not in the vessel.

\section{ANALYSIS CASES}

Structural analysis cases are presented for six model / load conditions which are summarized in Table I. In some observed halo asymmetries it appeared that the halo current varied around the torus with a peak to average current ratio of two. The $(1-\cos \theta)$ distribution $(\theta$ being the angular position around the machine axis) has that characteristic and was the basis for the first four cases. Only the second case, DT28, used the double wall model; it corresponds to single shell model case ST28 and used the ST28 blanket support forces as the asymmetry load. Case SH28 differs from ST28 in that it employs the hanging vessel support design.

Table I. Asymmetry Analysis Cases

\begin{tabular}{lcccc}
\hline $\begin{array}{l}\text { Case } \\
\text { Name }\end{array}$ & $\begin{array}{c}\text { Model } \\
\text { Type }\end{array}$ & $\begin{array}{c}\text { Support } \\
\text { Type }\end{array}$ & $\begin{array}{c}\text { Lateral } \\
\text { Load [MN] }\end{array}$ & $\begin{array}{c}\text { Halo Current } \\
\text { Distribution }\end{array}$ \\
\hline ST28 & $\begin{array}{c}\text { Single } \\
\text { Shell }\end{array}$ & Truss & 28 & $1-\cos \theta$ \\
DT28 & $\begin{array}{c}\text { Double } \\
\text { Wall }\end{array}$ & Truss & 28 & $1-\cos \theta$ \\
SH28 & $\begin{array}{c}\text { Single } \\
\text { Shell }\end{array}$ & Hanging & 28 & $1-\cos \theta$ \\
SH50A & $\begin{array}{c}\text { Single } \\
\text { Shell }\end{array}$ & Hanging & 50 & $1-\cos \theta$ \\
SH50B & $\begin{array}{c}\text { Single } \\
\text { shell }\end{array}$ & Hanging & 50 & $\begin{array}{c}\text { In bkt. 1- } \cos \theta \\
\text { Out bkt. 1+cos } \\
\text { Fx, Fy only: }\end{array}$ \\
SH50C & $\begin{array}{c}\text { Single } \\
\text { Shell }\end{array}$ & Hanging & 50 & $\begin{array}{c}\text { In bkt. 1- } \cos \theta \\
\text { Out bkt. 1+cos }\end{array}$ \\
\hline
\end{tabular}

The $(1-\cos \theta)$ distribution on this particular load set results in a net lateral load on the torus of $28 \mathrm{MN}$. However, recent guidelines from the ITER Joint Central Team (JCT) specified $50 \mathrm{MN}$ for lateral load due to halo asymmetry. The $(1-\cos \theta)$ distribution was additionally scaled by $50 / 28$ in SH50A to meet this guideline.

Other observations from experiments inferred that the plasma moves and / or tilts such that on one side of the torus the inner blanket sees a large halo current, while on the opposite side of the torus the outer blanket has halo current. To mimic this type of asymmetry, the poloidal current load group was further separated into inboard and outboard blanket sets which were separately scaled by $(1-\cos \theta)$ and $(1+\cos \theta)$. An additional overall scale factor was applied to obtain $50 \mathrm{MN}$ lateral load; vessel loads were left symmetric for these last two cases. In SH50C, vertical blanket forces were also excluded from scaling to also meet the JCT requirement for $150 \mathrm{MN}$ vertical load.

\section{RESULTS}

Elevation views of the deformed structure for the first three cases are shown in Figs. 4 through 6; a plan view of case $\mathrm{SH} 28$ is shown in Fig. 7. For reference, model segments are numbered 1 through 20 , with segment 1 being at $\theta=0^{\circ}$ and segment 11 at $180^{\circ}$. The origin is shown by cross hairs in each figure. Maximum total deformation in $\mathrm{mm}$ is indicated on each segment. Table II summarizes vessel inner leg lateral displacement (at the equatorial plane). Lateral displacement is generally constant between segments but vertical displacement varies around the torus, inferring both tipping and side movement. Comparing like models with different supports (ST28 to SH28), the truss support performs poorly in lateral constraint.

The higher displacement of the double wall model (DT28) was determined to be caused by lower toroidal stiffness in the vessel support attachment region. There was more local deformation here around segments 6 and 16 compared to the single shell case ST28. This discrepancy between single and double wall models had not been observed in symmetric analyses.

Table II. Vessel Lateral Displacement [mm] \& Support Reaction Forces [MN]

\begin{tabular}{lcccc}
\hline Case & $\begin{array}{c}\text { Vessel Lateral } \\
\text { Disp.* }\end{array}$ & $\begin{array}{c}\text { Total } \\
\text { Vertical react. }\end{array}$ & $\begin{array}{c}\text { Seg. 11 } \\
\text { Vertical react. }\end{array}$ & $\begin{array}{c}\text { Seg. 6/16 } \\
\text { Toroidal react. }\end{array}$ \\
\hline ST28 & 21 & 151 & 21 & 2.8 \\
DT28 & 32 & 151 & 24 & 4.8 \\
SH28 & 3.1 & 151 & 14 & 2.9 \\
SH50A & 5.4 & 239 & 24 & 5.1 \\
SH50B & 7.9 & 131 & 7.4 & 4.6 \\
SH50C & 8.1 & 151 & 7.9 & 4.6 \\
\hline
\end{tabular}

* Vessel inner leg lateral displacement on equatorial plane

Lateral loading is reacted through combination of toroidal and vertical forces at vessel support points which vary segment to segment; radial support reactions are negligible. Maximum reactions are also summarized in Table II. The differences between cases ST28 and DT28 are again attributed to lower toroidal stiffness in DT28. Results from case SH50A essentially scale up from $\mathrm{SH} 28$ by the increased loading, i.e., 50/28. Hanging support cases have less vertical force peaking as seen by segment 11 reaction force being closer to the average reaction per segment. The alternate distribution cases SH50B and SH50C have nearly uniform vertical reactions. Toroidal reactions for hanging support are at the equatorial port and induce bending stress at the port connection to the vessel. 


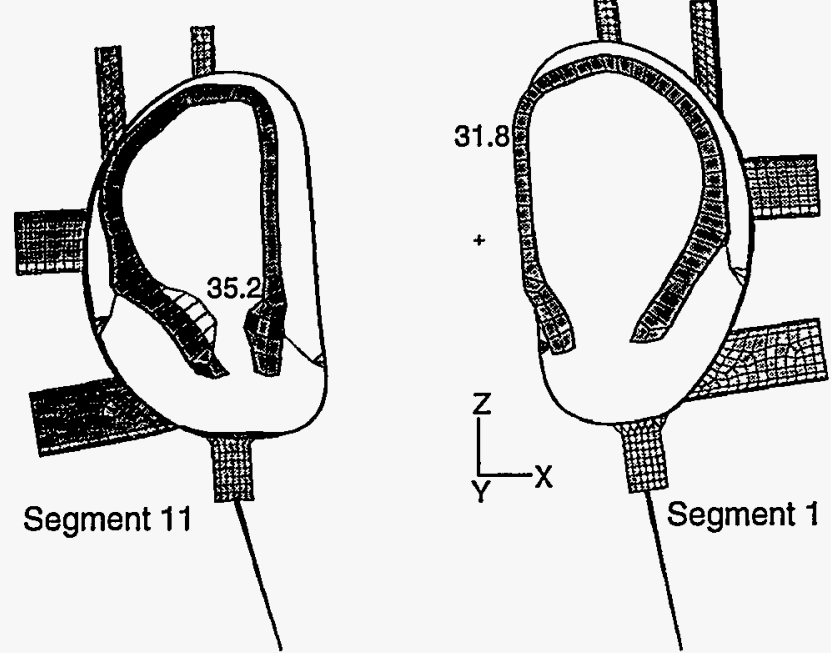

Fig. 4 Deformed Shape (100x) of Case ST28

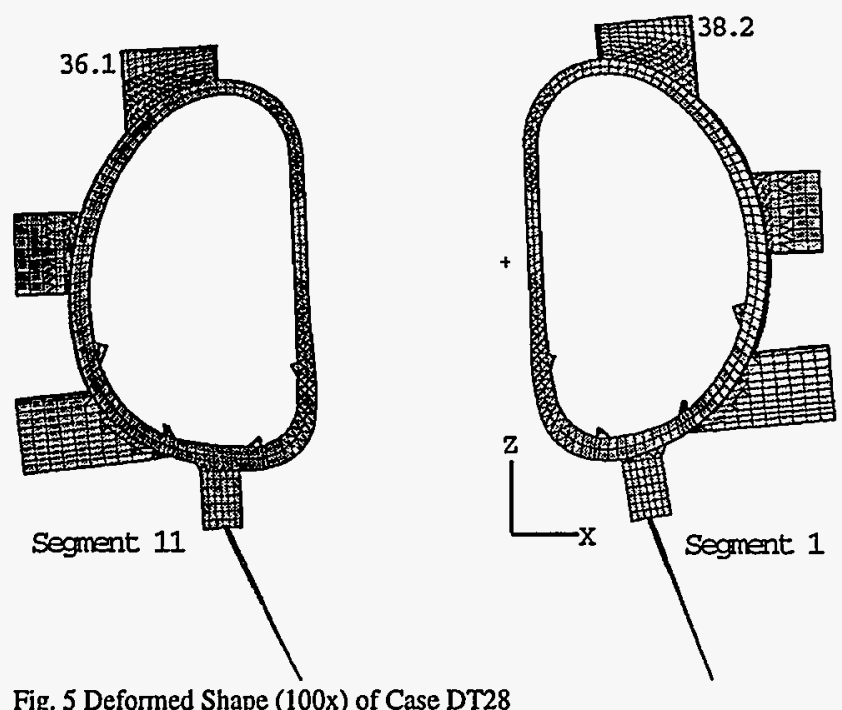

Fig. 5 Deformed Shape (100x) of Case DT28
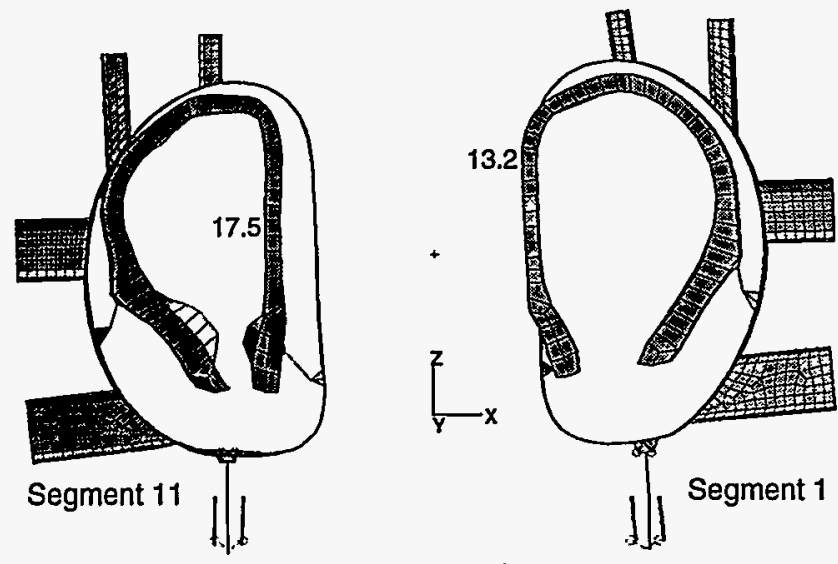

Fig. 6 Deformed Shape (100x) of Case SH28

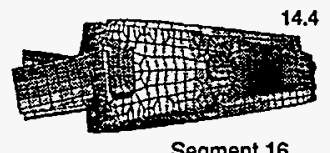

Segment 16

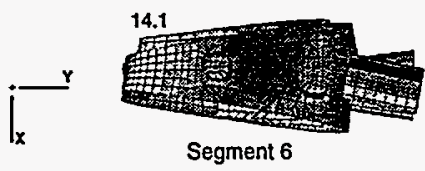

Fig. 7 Deformed Shape (100x) of Case SH28, Plan View

The base TSC load being used matches the JCT specification for vertical load. The $(1-\cos \theta)$ distribution does not alter total vertical load, but the additional scaling in SH50A to achieve $50 \mathrm{MN}$ lateral load had the adverse consequence of increasing vertical load to $239 \mathrm{MN}$. Conversely, the distribution applied to the blankets in case SH50B caused vertical load to fall below $150 \mathrm{MN}$. These were the factors which led to case SH50C in which vertical forces were completely excluded from asymmetry scaling.

Maximum vessel stresses in segments 1, 6,11 and 16 are given in Table III. Comparison of case SH28 to ST28 again shows the hanging vessel support to have an advantage over the truss. Maximum stress in single shell model cases generally occurs in the inner leg of the vessel just below the blanket attachment region. Noted exceptions to this are cases SH50B and SH50C, where in segments 6 and 16 it occurs in the equatorial port at the junction with the vessel. In these cases vertical leg stress is significantly lower than in SH50A. Double wall maximum stress occurs in the lower vessel support attachment region. Here there is a transition in the number of ribs between vessel walls and the indirect load path between them causes high bending stress.

Table III. Maximum Vessel Stress [MPa]

\begin{tabular}{lcccc}
\hline Case & Segment 1 & Segment 6 & Segment 11 & Segment 16 \\
\hline ST28 & 63 & 114 & 178 & 117 \\
DT28 & 109 & 240 & 231 & 250 \\
SH28 & 63 & 104 & 157 & 107 \\
SH50A & 104 & 179 & 267 & 186 \\
SH50B & 62 & 94 & 85 & 97 \\
SH50C & 69 & 94 & 90 & 97 \\
\hline
\end{tabular}

Maximum vessel stress estimated with symmetric disruption loading is considerably lower than results reported here. Unfortunately the only cases available for comparison also included simultaneous gravity and disruption loads. Nevertheless, in a static analysis of the ST28 model configuration with the peak TSC VDE load at $1.626 \mathrm{~s}$, maximum vessel stress was $107 \mathrm{MPa}$; dynamic analysis of the full VDE simulation gave only slightly higher stress of 112 $\mathrm{MPa}$. Dynamic analysis of the SH28 model gave an estimated vessel stress of $141 \mathrm{MPa}$.

Blanket support forces and moments vary from segment to segment depending on asymmetry distribution as well as 
stiffness of the supports, blankets and vessel. To illustrate, forces and bending moments from case SH28 are given in Fig. 8 for one of the inboard blanket supports. In these models there are two inboard and two outboard supports per segment. Blanket supports are the flexible plate assembly type and were modeled with beam and spring elements to provide equivalent stiffness.

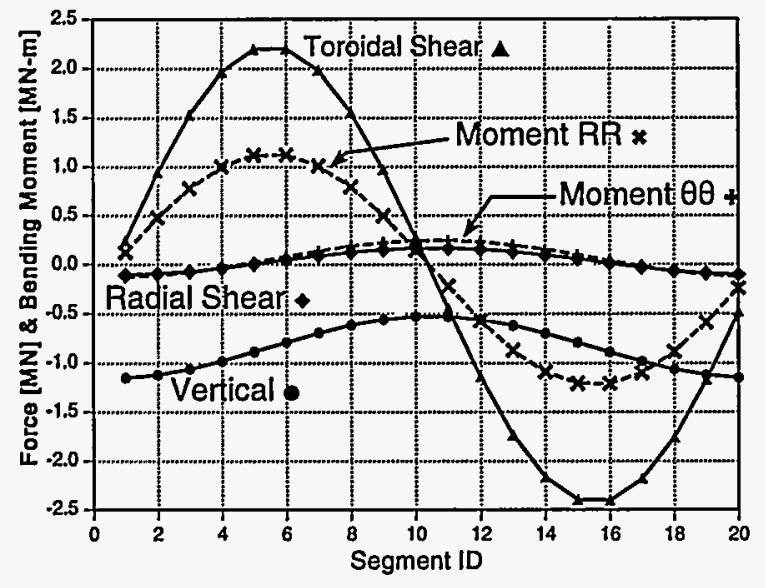

Fig. 8 Inboard Blanket Support Forces \& Moments, Case SH28

Forces and moments vary similarly between segments in cases ST28 and SH50A, although magnitudes differ more in the latter case. In SH50A, maximum vertical compression reaches -4.1 vs. $-1.2 \mathrm{MN}$ in $\mathrm{SH} 28$, and in both cases this occurs in segment 1 where the asymmetry scaling is zero. Outboard support vertical force reaches maximum compression in segment 11 , i.e, in sync with the scaling. Most of the lateral loading is being transmitted via support toroidal shear force and bending moment about local radial axes around segments 6 and 16. These are much lower under symmetric loading. For example, in the aforementioned dynamic analysis of the hanging support model, the inboard blanket support toroidal shear force is less than $0.15 \mathrm{MN}$ and the moment about the radial axis is less than $0.08 \mathrm{MN}-\mathrm{m}$.

Blanket support force variations in cases SH50B and $\mathrm{SH} 50 \mathrm{C}$, with their alternate asymmetries, differ from the other cases in several respects. Both inboard and outboard vertical support forces reach maximum compression in segment 11 and are lower in magnitude than the other cases. Shear forces and bending moments are lower as well; generally they are more uniform segment to segment and inboard to outboard. While these asymmetry conditions still result in net lateral load of $50 \mathrm{MN}$ on the torus, the forces are redistributed within the blanket resulting in more even loading on the torus compared to the $(1-\cos \theta)$ distribution cases. What this does to the blankets was not explored.

\section{CONCLUSIONS}

Static analyses of VDE load with postulated halo current asymmetries show significant lateral movement of the vacuum vessel torus and blankets not seen in analyses of symmetric disruption loading. Vessel stress is considerably higher under the asymmetric conditions.

The vessel support design which hangs the vessel from TF structure and provides a toroidal constraint at each equatorial port has significant advantages over the truss type vessel support in terms of lateral deflection and vessel stress.

Double wall model results, which employed blanket support forces from a corresponding single shell model, showed some inconsistencies with the single shell results that had not been observed in symmetric analyses. Regardless, double wall model maximum stress is being driven by poor load path in the rib transition region at the base of the vessel.

The alternate asymmetry conditions employed in two cases have relatively low vessel stress and blanket support forces for the same amount of lateral load. These asymmetry conditions distributed the lateral load in a manner more favorable to the vessel, but possibly worse for the blankets.

These disruption asymmetry conditions are potential design drivers since they result in high vessel stress and load the blankets and supports more severely than symmetric disruptions. Given the variations seen in results with the different conditions employed and considering that the asymmetry conditions used did not have robust bases, the need for better understanding of asymmetric disruptions and tools for estimating their loads becomes evident.

Including dynamic effects in asymmetric disruptions will present additional structural analysis difficulties. However, the effects may significantly impact the ITER design. Dynamic structural analysis of asymmetric disruption loading is an objective of future work.

\section{REFERENCES}

1. S. C. Jardin, N. Pomphrey, and J. Delucia, "Dynamic Modeling of Transport and Positional Control of Tokamaks", J. Comp. Physics 66, 481 (1986)

2. J. P. Caffrey, J. M. Lee, "MSC/NASTRAN Linear Static Analysis User's Guide, Version 68", The Macneal-Schwendler Corporation, 1994

3. "ITER Vacuum Vessel Dynamic Structural Analysis for Disruption Loading, Task D11", B. W. Riemer, ITER/US/94/IV-VV-05, ITER U.S. Home Team Report, January 1995

4. "ITER Vacuum Vessel Structural Analyses, CY1995", B. W. Riemer, ITER/US/95/INV-VV-04, ITER U.S. Home Team Report, May 1996

5. "TSC VDE Disruption Simulations for ITER-EDA", R. O. Sayer, ITER/US/94/INV-VV-02, ITER U.S. Home Team Report, Dec. 1994 


\section{DISCLAIMER}

This report was prepared as an account of work sponsored by an agency of the United States Government. Neither the United States Government nor any agency thereof, nor any of their employees, makes any warranty, express or implied, or assumes any legal liability or responsibility for the accuracy, completeness, or usefulness of any information, apparatus, product, or process disclosed, or represents that its use would not infringe privately owned rights. Reference herein to any specific commercial product, process, or service by trade name, trademark, manufacturer, or otherwise does not necessarily constitute or imply its endorsement, recommendation, or favoring by the United States Government or any agency thereof. The views and opinions of authors expressed herein do not necessarily state or reflect those of the United States Government or any agency thereof. 